\title{
Why is renal impairment associated with poorer cancer specific survival in breast cancer patients?: a comparison with patients with other comorbidities
}

\author{
Andy Evans $^{1}\left[\right.$ Russell Petty ${ }^{2} \cdot$ Jane Macaskill ${ }^{3}$
}

Received: 5 May 2020 / Accepted: 21 June 2020 / Published online: 2 July 2020

(c) The Author(s) 2020

\begin{abstract}
Background Our aim is to assess whether the poor breast cancer specific survival (BCSS) seen in women with breast cancer and impaired renal function can be explained by associations with other prognostic factors.

Methods The study group was a consecutive series of patients undergoing breast ultrasound (US) who had invasive breast cancer $(n=1171)$. All women had their US diameter and mean stiffness $(\mathrm{kPa})$ at shear wave elastography (SWE) recorded. The core biopsy grade and receptor status were noted. Core biopsy of abnormal axillary nodes and the patient referral source was also noted. Survival including cause of death was ascertained. Comorbidities at diagnosis were recorded. Patients were divided into those with a GFR $<60$ ("renal group"), those with other comorbidities and those with none. BCSS was assessed using Kaplan-Meier survival curves and Cox proportional hazards regression.

Results One thousand, one hundred and forty-one patients constituted the study group. 107 (9\%) patients had impaired renal function, 182 (16\%) had other comorbidities while 852 (75\%) had no comorbidities. Mean follow-up was 5.8 years. 109 breast cancer and 122 non-breast cancer deaths occurred. BCSS in the renal group was significantly worse than the other groups. Women with renal comorbidity were older, more likely to present symptomatically, have a pre-operative diagnosis of axillary metastases, and have larger and stiffer cancers. Cox proportional hazards regression showed that renal impairment maintained independent significance.

Conclusion The poor BCSS in women with impaired renal function is partially explained by advanced tumour stage at presentation. However, impaired renal function maintains an independent prognostic effect.
\end{abstract}

Keywords Renal impairment $\cdot$ Breast cancer $\cdot$ Mortality $\cdot$ Prognostic factors

Andy Evans

a.z.evans@dundee.ac.uk

Russell Petty

r.petty@dundee.ac.uk

Jane Macaskill

ejanemacaskill@nhs.net

1 Breast Imaging Group, Ninewells Hospital and Medical School, Mailbox 4, Dundee DD1 9SY, Scotland

2 Medical Oncology, Ninewells Hospital and Medical School, Mailbox 4, Dundee DD1 9SY, Scotland

3 Department of Breast Surgery, Ninewells Hospital, Level 6, Dundee DD1 9SY, Scotland

\section{Introduction}

Comorbidities are important in breast cancer management as many women with breast cancer die from other causes and comorbidities impact on treatment options for breast cancer patients, particularly the appropriateness of chemotherapy.

There is a linear decline in glomerular filtration rate (GFR) from age 30 so that values in octagenarians are only half to two-thirds of those measured in young adults [1]. As breast cancer incidence increases with age, it is therefore common to find older women presenting with breast cancer and impaired renal function. While many comorbidities are associated with poorer overall survival in women with breast cancer [2,3], a number of studies have found that impaired renal function is associated with poorer breast cancer specific survival $[4,5]$ while other comorbidities such as diabetes and cerebro-vascular disease are not $[5,6]$. 
Little attention has previously been paid to correcting for known tumour prognostic factors when evaluating the impact of comorbidities on breast cancer survival. However, patients with comorbidities are more likely to receive primary or neoadjuvant endocrine therapy and not receive immediate surgery, the specimen from which has traditionally been used to assess prognosis. Therefore, traditional prognostic information is not available in many women with comorbidities. However, recent work has highlighted prognostic indicators which are available pre-operatively on all patients. These include tumour core biopsy grade, oestrogen (ER) and HER-2 receptor status, ultrasound (US) tumour size, pre-operative diagnosis of axillary metastases, patient source, (mammographic screening or symptomatic presentation [7]) and lesion stiffness on shear wave elastography (SWE) which is an ultrasound technique [8].

The aim of this study is to assess whether the poor prognostic effect of impaired renal function can be explained by associations with other known prognostic factors or if impaired renal function has independent prognostic significance in a prospectively collected consecutive series of patients with invasive breast cancer.

\section{Materials and methods}

The study group was collected prospectively from a consecutive series of patients undergoing diagnostic breast US examination for lesions subsequently shown to be invasive breast cancer $(n=1171)$ between April 2010 and September 2015. All women had their US lesion diameter and mean stiffness $(\mathrm{kPa})$ at SWE recorded irrespective of subsequent treatment (primary surgery, neoadjuvant systemic therapy and primary endocrine therapy). The core biopsy grade was recorded and ER status and HER-2 status were measured on the core biopsy samples. All women had axillary US and core biopsy of abnormal nodes (node cortical thickness of $>2.3 \mathrm{~mm}$ was considered abnormal) for assessment of nodal status. The patient source, (mammographic screening or symptomatic presentation) was also recorded.

Patient's survival including cause of death was ascertained from local paper and electronic health records and the National Cancer Registry. Patients who died after developing metastatic breast cancer were assumed to have died of breast cancer. A total of 30 patients were excluded from the analysis on the following grounds: metastases at presentation; cause of death that could not be ascertained and history of a previous breast cancer.

Comorbidities included in the Charlson comorbidity index [9] were obtained retrospectively by examining the local electronic health records prior to diagnosis. Factors recorded included a GFR less than 60, diabetes, liver failure, previous non-breast invasive cancer (excluding non-melanoma skin cancer), lymphoma, multiple sclerosis, peripheral vascular disease, stroke/TIA, coronary heart disease, ulcerative colitis, chronic obstructive pulmonary disease (COPD), dementia, SLE, peptic ulcer and rheumatoid arthritis.

Patients were divided into three groups: those with a GFR $<60$ (renal group), those with other comorbidities and those with no comorbidities. If patient had multiple comorbidities including reduced renal function, they were included in the renal comorbidities group.

The non-breast cancer mortality of the renal comorbidity group, the other comorbidity group and the no comorbidity group was assessed using Kaplan-Meier survival curves. The differences between categorical cancer and patient characteristics in the renal and other comorbidity groups were assessed using the Chi-square and Fisher's exact test. Continuous characteristics were assessed using a comparison of ROC curves using the log-rank test. Kaplan-Meier survival curves were also used to compare the BCSS of the renal and other comorbidity groups. Cox proportional hazards regression was used to assess the independent impact on breast cancer specific survival of renal impairment. This analysis included factors found to be significant on univariate analysis when comparing the renal and other comorbidity groups and was carried out on a combined dataset of both these groups. Statistical analyses were performed using Med Calc software.

\section{Results}

After the exclusions detailed above, 1141 patients constituted the study group. 107 (9\%) patients had impaired renal function, $182(16 \%)$ had other comorbidities with normal renal function while 852 (75\%) had no comorbidities. Of the 107 patients with impaired renal function 45 had other comorbidities, most commonly diabetes $(n=21)$. The mean age of patients in the renal, other and no comorbidity groups were 75.3 years, 69.0 years and 59.8 years, respectively. Table 1 shows the frequency of comorbidities. The

Table 1 Frequency of commonest comorbidities

\begin{tabular}{lc}
\hline Comorbidity & Number (\%) \\
\hline Renal impairment & $107(9 \%)$ \\
Diabetes & $70(6 \%)$ \\
Coronary heart disease & $31(3 \%)$ \\
Other malignancy & $30(3 \%)$ \\
COPD & $25(2 \%)$ \\
Cerebrovascular disease & $21(2 \%)$ \\
Peptic ulcer & $19(2 \%)$ \\
Rheumatoid arthritis & $18(2 \%)$ \\
Dementia & $8(1 \%)$ \\
Peripheral vascular disease & $4(0.4 \%)$ \\
\hline
\end{tabular}




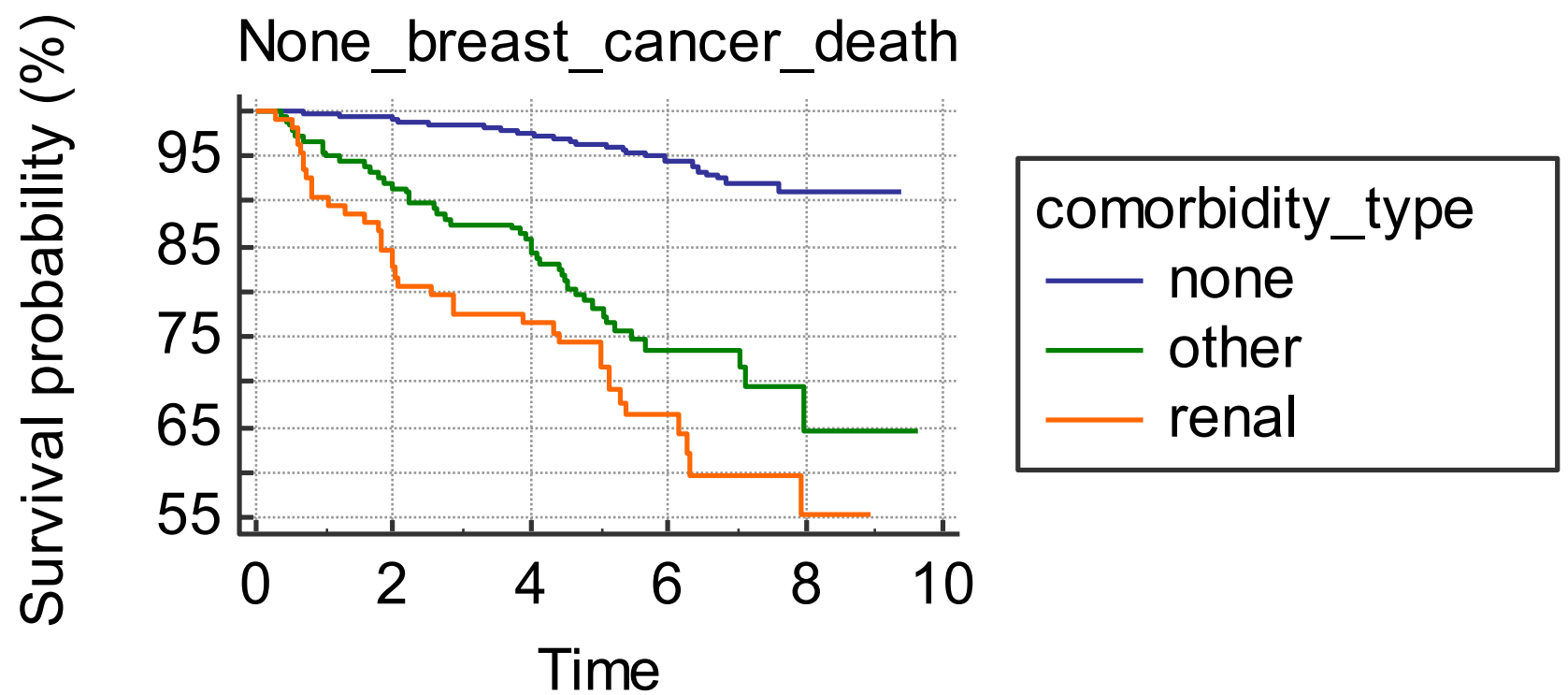

Number at risk

Group: none

$\begin{array}{llllll}849 & 817 & 664 & 321 & 62 & 0\end{array}$

Group: other

$\begin{array}{llllll}178 & 161 & 136 & 61 & 13 & 0\end{array}$

Group: renal

$\begin{array}{llllll}107 & 83 & 72 & 32 & 12 & 0\end{array}$

Comparison of survival curves (Logrank test)

\begin{tabular}{|l|r|}
\hline Chi-squared & 128.1444 \\
\hline DF & 2 \\
\hline Significance & $\mathrm{P}<0.0001$ \\
\hline
\end{tabular}

Hazard ratios ${ }^{a}$ with $95 \%$ Confidence Interval

\begin{tabular}{|l|c|c|c|}
\hline Factor & none & other & renal \\
\hline none & - & 5.2412 & 7.8357 \\
& 0.1908 & 3.1870 to 8.6195 & 4.1045 to 14.9589 \\
\hline other & 0.1160 to 0.3138 & - & 1.4950 \\
& 0.1276 & 0.6689 & 0.6966 to 3.2084 \\
renal & 0.06685 to 0.2436 & 0.3117 to 1.4355 & - \\
\hline
\end{tabular}

Fig. 1 Kaplan-Meier survival curves for non-breast cancer deaths according to type and presence of comorbidities 


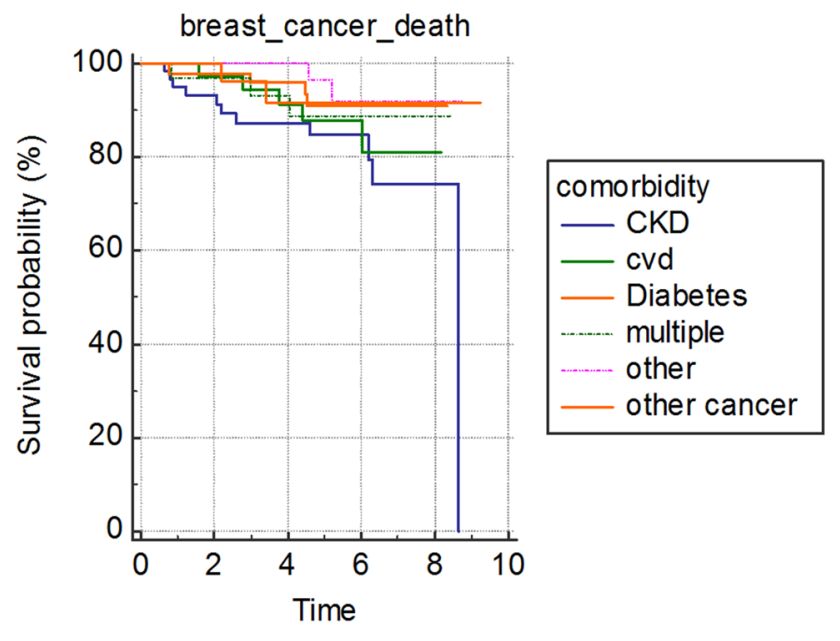

Fig. 2 Breast cancer survival for patients with individual comorbidities

commonest comorbidities were impaired renal function followed by diabetes.

Mean follow-up in those alive at the end of follow-up was 5.8 years. One hundred and nine breast cancer and 122 non-breast cancer deaths occurred in the entire cohort. The number of breast cancer and non-breast cancer deaths in the renal comorbidity group was 18 and 36 , respectively. The number of breast cancer and non-breast cancer deaths in the other comorbidity group was 11 and 44 , respectively.

Figure 1 shows the non-breast cancer mortality for the three groups. The non-breast cancer mortality in the two comorbidity groups was significantly worse than in the no comorbidity group $(<0.0001)$. However, the non-breast cancer mortality was similar in the renal and other comorbidity groups.

Figure 2 shows the breast cancer mortality of the individual comorbidities. The curves suggest that patients with impaired renal function have poorer breast cancer specific survival than patients with other individual comorbidities.

Figure 3 shows the breast cancer mortality for the three groups. The breast cancer mortality in the renal group was significantly worse than either the other comorbidity group or the no comorbidity group.

Table 2 shows the breast cancer and patient characteristics of the two comorbidity groups. Women with renal comorbidity were older and more likely to have symptomatic cancer than women in the other comorbidity group. Cancers in the renal group were more likely to have a pre-operative diagnosis of axillary metastases, had larger US sizes, and were stiffer on SWE.
The results of Cox proportional hazards regression for breast cancer death in the renal comorbidity and other comorbidity groups combined are shown in Table 3 . The analysis included those factors shown to be significant when comparing the renal and other comorbidity groups, i.e., age, source (symptomatic or screening presentation), ultrasound size, stiffness at SWE and pre-operative diagnosis of axillary metastases. The strongest predictor of breast cancer death was a preoperative diagnosis of axillary metastases, but renal impairment maintains independent significance. Ultrasound tumour size and patient source were of borderline significance.

\section{Discussion}

We have shown that that the poorer breast cancer specific survival in patients with impaired renal function is related to more advanced tumour stage at diagnosis, but that impaired renal function is still an independent poor prognostic marker. The more advanced stage in these patients may be related to the patient's age, as most are above routine screening age and present with symptoms. Breast cancer patients with impaired renal function are significantly older than patients with other comorbidities. Older patients with comorbidities might also be more reluctant to present or present later with breast symptoms than younger women with comorbidities.

There have been a number of previous suggestions as to why impaired renal function is associated with poor breast cancer survival, including that renal impairment leads to the development of more aggressive, or more treatmentresistant disease [10]. This would seem unlikely as the histological grade distribution and ER and HER-2 receptor status of patients with renal and other comorbidities are similar (Table 2). The suggestion that comorbidity may lead to poor organ function, making it difficult to receive optimal therapy and therefore suboptimal treatment seems credible. Chemotherapy rates were low in both comorbidity groups in our study but lowest in the renal group (13\% vs 19\%). Despite a plan to give chemotherapy, poor renal function may lead to dose reduction or discontinuation more commonly than in women with non-renal comorbidities or without comorbidity. Approximately half of all chemotherapy agent are excreted the kidneys, so poor renal function can lead to accumulation of toxic metabolites and overdosage [11]. Reduced renal function is also associated with a higher risk of developing cardiotoxicity in patients with HER 2 positive disease being treated with Trastuzumab [12]. It has been suggested that erythropoiesis stimulating agents may 


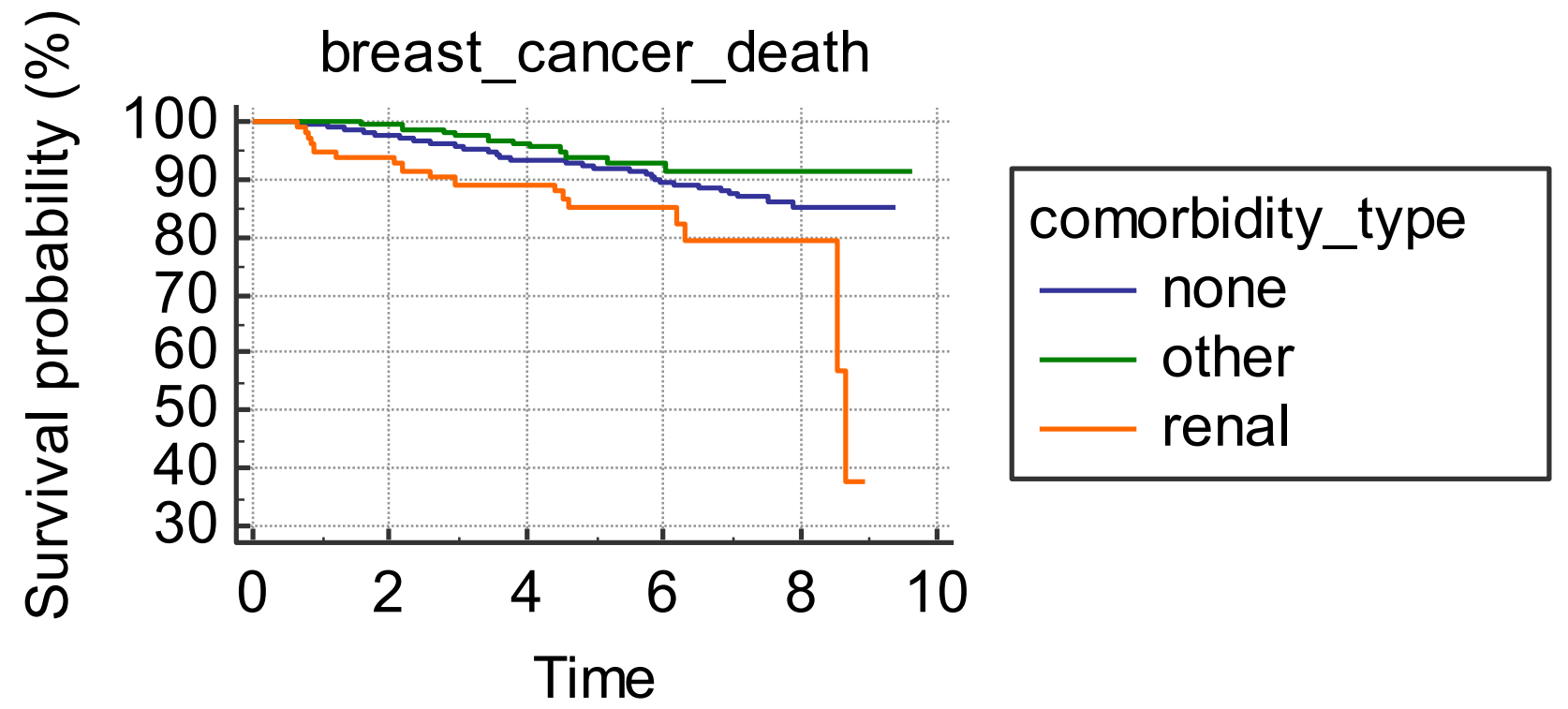

Number at risk

Group: none

$\begin{array}{llllll}849 & 817 & 664 & 321 & 62 & 0\end{array}$

Group: other

$\begin{array}{llllll}178 & 161 & 136 & 61 & 13 & 0\end{array}$

Group: renal

$\begin{array}{llllll}107 & 83 & 72 & 32 & 12 & 0\end{array}$

Comparison of survival curves (Logrank test)

\begin{tabular}{|l|r|}
\hline Chi-squared & 10.8155 \\
\hline DF & 2 \\
\hline Significance & $P=0.0045$ \\
\hline
\end{tabular}

Hazard ratios ${ }^{a}$ with $95 \%$ Confidence Interval

\begin{tabular}{|c|c|c|c|}
\hline Factor & none & other & renal \\
\hline none & - & $\begin{array}{c}0.6674 \\
0.3963 \text { to } 1.1240\end{array}$ & $\begin{array}{c}2.0324 \\
1.0273 \text { to } 4.0212\end{array}$ \\
\hline other & $\begin{array}{c}1.4983 \\
0.8897 \text { to } 2.5232\end{array}$ & - & $\begin{array}{c}3.0452 \\
1.3645 \text { to } 6.7960\end{array}$ \\
\hline renal & $\begin{array}{c}0.4920 \\
0.2487 \text { to } 0.9735\end{array}$ & $\begin{array}{c}0.3284 \\
0.1471 \text { to } 0.7329\end{array}$ & - \\
\hline
\end{tabular}

Fig. 3 Kaplan-Meier survival curves for breast cancer deaths according to type and presence of comorbidities 
Table 2 Tumour and patient characteristics of the renal and other comorbidity groups

\begin{tabular}{lllll}
\hline & Renal & Other & None & Renal vs other \\
\hline Pre-op nodal mets & $23(21)$ & $21(12)$ & $151(18)$ & $P=0.02$ \\
Grade 1 & $8(7)$ & $22(12)$ & $109(13)$ & $P=0.49$ \\
Grade 2 & $66(62)$ & $101(55)$ & $455(53)$ & \\
Grade 3 & $33(31)$ & $59(32)$ & $288(34)$ & \\
Us size $<15$ & $33(31)$ & $80(44)$ & $393(46)$ & $P=0.027$ \\
Us size > or equal 15 & $74(69)$ & $102(56)$ & $459(54)$ & $P=0.006$ on \\
& & & & continuous \\
& & & & data \\
ER pos & $89(83)$ & $152(84)$ & $714(84)$ & $P=1$ \\
Er neg & $18(17)$ & $30(16)$ & $138(16)$ & \\
Her 2 pos & $12(12)$ & $16(9)$ & $109(14)$ & $P=0.48$ \\
Her 2 neg & $91(88)$ & $161(91)$ & $733(86)$ & \\
Screen detected & $24(22)$ & $62(34)$ & $347(41)$ & $P=0.037$ \\
Mean age & 75.4 & 69.0 & 59.8 & $P<0.0001$ \\
Chemo & $14(13)$ & $35(19)$ & $317(37)$ & $P=0.17$ \\
Mean stiffness at SWE & 140 & 120 & 130 & $P=0.004$ \\
Total & 107 & 182 & 852 & \\
\hline
\end{tabular}

be associated with tumour progression [13]; however, these agents were not routinely used in women having chemotherapy in this cohort and the chemotherapy rate itself was low. Chemotherapy use was not included in the proportional hazards regression as it was not significant in the univariate analysis comparing the renal and other comorbidity groups.

Smoking causes reno-vascular disease and many types of cancer but is only weakly associated with breast cancer [14] and poorer breast cancer specific survival [15]; thus, previous postulated causation of smoking is unlikely to be an important mechanism in this instance. We did not have access to smoking history in our cohort.
Despite the poor breast cancer specific survival in the renal comorbidity group, it should be remembered that twice as many patients died from non-breast cancer causes during follow-up. This is in comparison with a 4:1 ratio of non-breast cancer deaths to breast cancer deaths in the other comorbidity group. It is therefore important that potential treatment toxicity issues with systemic therapy are taken seriously in all patients with comorbidities.

Weaknesses of the study include it being from a single centre and that despite the initial large cohort size, the number of breast cancer deaths in women with impaired renal function is modest. The study looked only at baseline renal function which of course changes over time. This study did not look at proteinuria as this was not routinely available in this cohort. The strength of this study lies in attempting to ascertain whether tumour and patient factors contribute to or are solely responsible for the poor breast cancer survival in women with impaired renal function.

\section{Conclusions}

The poor breast cancer specific survival in women with impaired renal function is partially explained by advanced tumour stage at presentation. However impaired renal function maintains an independent poor prognostic effect even when tumour factors and patient age are taken into account.

Funding This research did not receive any specific grant from funding agencies in the public, commercial, or not-for-profit sectors.

\section{Compliance with ethical standards}

Conflict of interest The authors have no conflict of interest.
Table 3 Cox proportional hazards regression for breast cancer death in women with renal and other comorbidities

\begin{tabular}{lllllll}
\hline Covariate & $b$ & SE & Wald & $P$ & $\operatorname{Exp}(b)$ & 95\% CI of Exp $(b)$ \\
\hline Age & 0.01531 & 0.01646 & 0.8654 & 0.3522 & 1.0154 & $0.9832-1.0487$ \\
Stiffness & 0.0004727 & 0.003075 & 0.02363 & 0.8778 & 1.0005 & $0.9945-1.0065$ \\
Us_size & 0.03746 & 0.01970 & 3.6153 & 0.0572 & 1.0382 & $0.9988-1.0790$ \\
Preop_nodal_mets & 1.4398 & 0.3874 & 13.8104 & 0.0002 & 4.2196 & $1.9747-9.0169$ \\
Renal impairment & 0.8425 & 0.3972 & 4.4985 & 0.0339 & 2.3221 & $1.0660-5.0583$ \\
Source & 1.0830 & 0.5679 & 3.6365 & 0.0565 & 2.9536 & $0.9703-8.9907$ \\
\hline
\end{tabular}


Open Access This article is licensed under a Creative Commons Attribution 4.0 International License, which permits use, sharing, adaptation, distribution and reproduction in any medium or format, as long as you give appropriate credit to the original author(s) and the source, provide a link to the Creative Commons licence, and indicate if changes were made. The images or other third party material in this article are included in the article's Creative Commons licence, unless indicated otherwise in a credit line to the material. If material is not included in the article's Creative Commons licence and your intended use is not permitted by statutory regulation or exceeds the permitted use, you will need to obtain permission directly from the copyright holder. To view a copy of this licence, visit http://creativecommons.org/licenses/by/4.0/.

\section{References}

1. Brenner BM, Meyer TW, Hostetter TH (1982) Dietary protein intake and the progressive nature of kidney disease: the role of hemodynamically mediated glomerular injury in the pathogenesis of progressive glomerular sclerosis in aging, renal ablation, and intrinsic renal disease. N Engl J Med 307(11):652-659

2. Parés-Badella $O$, Banquéa $M$, Maciàa $F$ et al (2017) Impact of comorbidity on survival by tumour location: breast, colorectal and lung cancer (2000-2014). Cancer Epidemiol 49:66-74

3. Patnaik J, Byers T, DiGuiseppi C et al (2011) The influence of comorbidities on overall survival among older women diagnosed with breast cancer. J Natl Cancer Inst 103:1101-1111

4. Iff S, Craig JC, Turner R et al (2014) Reduced estimated GFR and cancer mortality. Am J Kidney Dis 63:23-30

5. Ewertza M, Holm Landa L, Daltonb S et al (2018) Influence of specific comorbidities on survival after early-stage breast cancer. Acta Oncol 57:129-134

6. Maskarinec G, Shvetsov Y, Conroy S et al (2019) 1Type 2 diabetes as a predictor of survival among breast cancer patients: the multiethnic cohort. Breast Can Res Treat 173:637-645

7. Puvanesarajah S, Gapstur S, Patel A et al (2019) Mode of detection and breast cancer mortality by follow-up time and tumor characteristics among screened women in Cancer Prevention Study-II. Breast Can Res Treat 177:679-689

8. Evans A, Sim YT, Pourreyron C et al (2018) Pre-operative stromal stiffness measured by shear wave elastography is independently associated with breast cancer-specific survival. Breast Cancer Res Treat 171(2):383-389

9. Quan H, Li B, Couris C et al (2011) Updating and validating the charlson comorbidity index and score for risk adjustment in hospital discharge abstracts using data from 6 countries. Am J Epidemiol 173:676-682

10. Kimmick G, Li X, Fleming S, Sabatino S et al (2018) Risk of cancer death by comorbidity severity and use of adjuvant chemotherapy among women with locoregional breast cancer. J Geriatr Oncol 9:214-220

11. Małyszko J, Kozlowski L, Kozłowska K et al (2017) Cancer and the kidney: dangereoux liasons or price paid for the progress in medicine? Oncotarget 8:66601-66619

12. Russo G, Cioffi G, Di Lenarda A et al (2012) Role of renal function on the development of cardiotoxicity associated with trastuzumab-based adjuvant chemotherapy for early breast cancer. Intern Emerg Med 7:439-446

13. Tonia T, Mettler A, Robert N et al (2012) Erythropoietin or darbepoetin for patients with cancer. Cochrane Database Syst Rev 12:CD003407

14. Jones M, Schoemaker MJ, Wright LB et al (2017) Smoking and risk of breast cancer in the Generations Study cohort. Breast Cancer Res 19:118

15. Passarelli MN, Newcomb PA, Hampton JM et al (2016) Cigarette smoking before and after breast cancer diagnosis: mortality from breast cancer and smoking-related diseases. J Clin Oncol 34(12):1315-1322

Publisher's Note Springer Nature remains neutral with regard to jurisdictional claims in published maps and institutional affiliations. 